\title{
Visceral adiposity would be expected to predict incident diabetes better in women than men
}

\author{
C. M. Schooling • C. Q. Jiang • W. S. Zhang • \\ T. H. Lam • K. K. Cheng • G. M. Leung
}

Received: 22 October 2009 / Accepted: 29 October 2009 /Published online: 24 November 2009

(C) Springer-Verlag 2009

Keywords Blood pressure - China - Diabetes - Glucose Muscle mass · Older $\cdot$ Waist-hip ratio

To the Editor: We read with great interest the report by Hanley et al. [1] that includes the observation that visceral adipose tissue predicted incident type 2 diabetes more strongly in women than in men. This unexplained observation comes from a large detailed well-designed prospective study in under-studied populations and, as the authors point out, it is also consistent with previous studies, suggesting that it is not a chance finding but may be of aetiological significance. In fact, this observation is to be expected from a life-course perspective. We have produced epidemiological evidence, gained using several different study designs in different populations, that is consistent with the hypothesis that nutritionally and inter-generationally driven levels of sex steroids at puberty play a role in cardiovascular diseases [2]. It is well known that sexually dimorphic

C. M. Schooling $\cdot$ T. H. Lam $(\bowtie) \cdot$ G. M. Leung

School of Public Health, Li Ka Shing Faculty of Medicine,

The University of Hong Kong, 21 Sassoon Road,

Pokfulam, Hong Kong SAR, People's Republic of China

e-mail: hrmrlth@hkucc.hku.hk

C. Q. Jiang · W. S. Zhang

Guangzhou Occupational Diseases Prevention and Treatment

Centre, Guangzhou Number 12 Hospital, Guangzhou,

People's Republic of China

K. K. Cheng

Department of Public Health and Epidemiology,

The University of Birmingham, Birmingham, UK body shapes, including less central obesity in women but more central obesity in men, become more pronounced at puberty because of the action of sex steroids [3], which also promote the accumulation of muscle mass [4]. As a consequence, diabetes should be more strongly associated with central obesity in women, in whom it reflects low muscle mass, than in men, in whom it does not. Furthermore, we would also expect this sex-specific association with central obesity to be specific to glucose metabolism rather than all cardiovascular risk factors, because there is no physiological reason to think that the same mechanism (muscle mass) impacts on factors such as blood pressure. To further test this hypothesis, we examined the associations of waist-hip ratio with fasting glucose and blood pressure, and whether the associations varied by sex, in a large sample of older individuals from south China.

The Guangzhou Biobank Cohort Study has been described in detail elsewhere [5]. In brief, recruitment-restricted to those aged at least 50 years - was carried out in three phases (approximately 10,000 participants per phase), from September 2003 to January 2008. The detailed methods of measurement have been reported [5]. At the half-day physical examination participants were asked about their use of medication. Waist circumference was measured horizontally around the smallest circumference between the ribs and iliac crest, or at the level of the navel for obese participants. Hip circumference was measured at the greatest circumference round the buttocks below the iliac crest. Fasting glucose was determined by the Shimadzu CL-8000 Clinical Chemical Analyzer (Shimadzu Corp., Kyoto, Japan). Seated blood pressure was recorded as the average of the last two of three measurements using the Omron 705CP sphygmomanometer (Omron Corp., Kyoto, Japan). The Guangzhou Medical Ethics Committee of the Chinese Medical Association 
approved the study and all participants gave written informed consent before participation.

To display the association between waist-hip ratio and fasting glucose in men and women, we used locally weighted scatterplot smoothing. To test whether waist-hip ratio had a different association with fasting glucose by sex we examined the significance of interaction terms from censored linear regression adjusted for age (in 5 year age bands). Censored regression is designed for data where some measurements are censored below (or above) the true values, such as by medication use. Unlike ordinary least squares estimates, censored estimates are consistent and efficient for censored data. We similarly examined the associations between waist-hip ratio and blood pressure (systolic and diastolic).

Figure 1 shows that fasting glucose increased more steeply with waist-hip ratio in women than in men. Figure 1 also shows that, in contrast, blood pressure increased more steeply with waist-hip ratio in men than in women. There was evidence that all associations differed in men and women (all $p$ values for interaction $<0.01$ ).

Consistent with the observations of Hanley et al. [1], we found in a large sample from an under-studied population
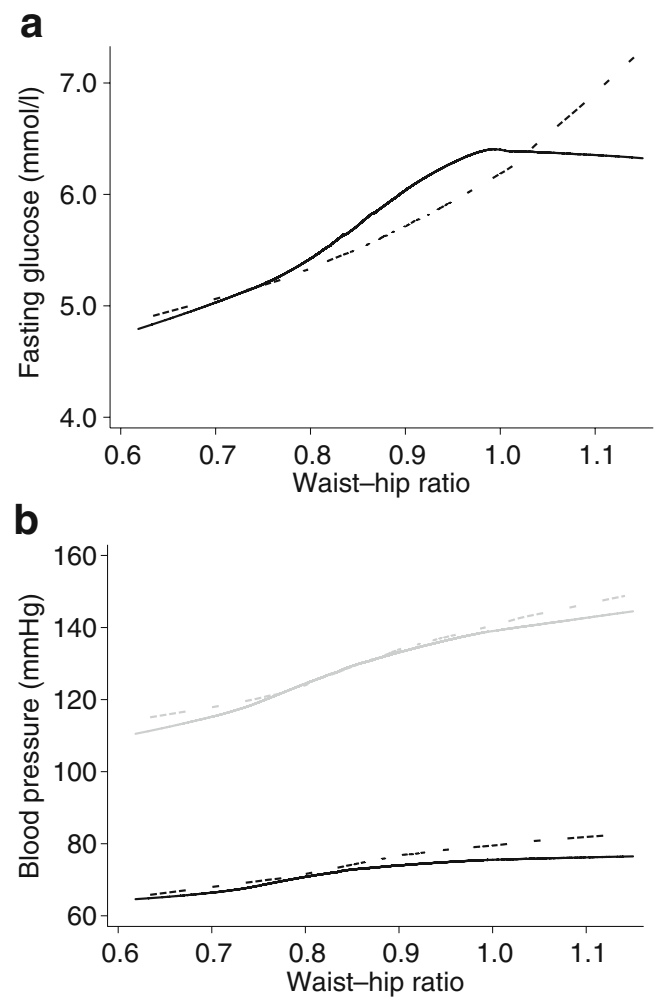

Fig. 1 a Relationship of fasting glucose with waist-hip ratio in men (dashed line) and women (solid line), b Relationship of systolic (grey lines) and diastolic (black lines) blood pressure with waist-hip ratio in men (dashed lines) and women (solid lines) from the Guangzhou Biobank Cohort Study (8,320 men and 21,806 women) that fasting glucose was more strongly associated with central obesity in women than in men. However, we have now added to this by showing that this association is specific to markers of diabetes and by providing a theoretically based explanation from a life-course perspective.

It is not our intention to discount other well-established aspects of the developmental origins of diabetes, such as fetal undernutrition, nor to discount the role of wellestablished risk factors. We are merely drawing attention to the long-term biological consequences of well-known sex-specific changes in body composition that take place at puberty, driven by sex steroids.

Notably, both Hanley et al. [1] and our group were examining populations other than Europeans living in long-term developed regions. It may be that pubertal development covers a wider range of exposures in such populations, and our observations only apply to those populations. However, this negates neither the importance of the potential mechanism, nor its implications for the rest of the world and the changing disease patterns it is experiencing. Economic development over generations may increase levels of sex steroids [6]. One key exposure (pubertal sex steroids) could provide a simple sociobiological explanation for the secular trends in diabetes and ischaemic heart disease that occur with economic development. These trends include relatively high diabetes rates in both sexes without commensurate ischaemic heart disease in men in populations with a short history of economic development - such as in Asians [7, 8] and those who have migrated to more economically developed countries from relatively less developed ones $[9,10]$ - and higher ischaemic heart disease among men in populations of long-term developed regions [8]. Moreover, from a public health perspective, adolescence may be a key phase when the risks for non-communicable disease associated with economic development are actuated and thus it may be a key window for intervention, perhaps by building muscle mass. Moreover, this tentative socio-biological hypothesis draws attention to history rather than geography; rather than looking to ethnic, cultural or genetic causes of disease, we should perhaps focus on mechanisms such as epigenetics, which may have 'long-range' effects over generations of economic development.

Acknowledgements The study was funded by: the University of Hong Kong Foundation for Development and Research and the University of Hong Kong University Research Committee Strategic Research Theme Public Health, Hong Kong; the Guangzhou Public Health Bureau and Guangzhou Science and Technology Bureau, Guangzhou, China; and the University of Birmingham, UK.

Duality of interest The authors declare that there is no duality of interest associated with this manuscript. 


\section{References}

1. Hanley AJ, Wagenknecht LE, Norris JM et al (2009) Insulin resistance, beta cell dysfunction and visceral adiposity as predictors of incident diabetes: the Insulin Resistance Atherosclerosis Study (IRAS) Family Study. Diabetologia 52:2079-2086

2. Chung RY, Schooling CM, Cowling BJ, Leung GM (2009) How does socioeconomic development affect risk of mortality? An ageperiod-cohort analysis from a recently transitioned population in China. Am J Epidemiol doi:10.1093/aje/kwp378

3. Loomba-Albrecht LA, Styne DM (2009) Effect of puberty on body composition. Curr Opin Endocrinol Diabetes Obes 16:10-15

4. Rico H, Revilla M, Villa LF, Hernandez ER, de Varez BM, Villa M (1993) Body composition in children and Tanner's stages: a study with dual-energy x-ray absorptiometry. Metabolism 42:967-970
5. Jiang C, Thomas GN, Lam TH et al (2006) Cohort profile: The Guangzhou Biobank Cohort Study, a Guangzhou-Hong KongBirmingham collaboration. Int J Epidemiol 35:844-852

6. Nunez-de la Mora A, Chatterton RT, Choudhury OA, Napolitano DA, Bentley GR (2007) Childhood conditions influence adult progesterone levels. PLoS Med 4:e167

7. Chan JC, Malik V, Jia W et al (2009) Diabetes in Asia: epidemiology, risk factors, and pathophysiology. JAMA 301:2129-2140

8. Lawlor DA, Ebrahim S, Smith GD (2001) Sex matters: secular and geographical trends in sex differences in coronary heart disease mortality. BMJ 323:541-545

9. Bolduan C, Weiner L (1933) Causes of death among Jews in New York City. N Engl J Med 208:407-416

10. Cowie CC, Rust KF, Ford ES et al (2009) Full accounting of diabetes and pre-diabetes in the U.S. population in 1988-1994 and 2005-2006. Diabetes Care 32:287-294 